\section{Is universal tumor testing for Lynch syndrome cost-effective? It depends!}

To the Editor

A newly published systematic review of cost-effectiveness analyses of testing strategies for Lynch syndrome (LS) by $\mathrm{Di}$ Marco et al. ${ }^{1}$ provides a useful summary of published estimates of incremental cost-effectiveness ratios (ICERs). Readers may wish to convert the reported ICER estimates into a single currency year (e.g., 2014 US dollars or euros), as was done in a previous review, ${ }^{2}$ to compare estimates across studies.

For readers to interpret published estimates of ICERs, it is important to keep in mind that the cost-effectiveness of an intervention, such as genetic testing for LS, is inherently a function of the comparator and context. ${ }^{2}$ A service may be cost-effective compared with doing nothing but not when compared with an alternative strategy. Second, value, like beauty, is in the eye of the beholder. The revealed preferences of payers (willingness to pay to cover a service) is itself a measure of cost-effectiveness. For many decision makers, considerations, such as perceptions of affordability (modest budget impact), fairness, administrative simplicity, and standard of care, may be more influential than ICERs in choosing among alternative strategies. Third, costeffectiveness estimates are inherently uncertain; a point ICER estimate that is below a given benchmark value may have a true value that is above the cutoff. Finally, because testing costs differ markedly between healthcare systems and over time, cost-effectiveness generalizations can be misleading. Ultimately, whether universal tumor testing is considered cost-effective relative to targeted testing can depend on the cost of tumor testing strategies, the numbers of relatives detected through cascade screening, and how much decision makers are willing to pay for modest improvements in case detection.

Di Marco et al. conclude that "from a health-care perspective, the cost-effectiveness of both universal and agetargeted CRC-based LS screening is acceptable in terms of willingness-to-pay for health gains". They are at least half right. Published studies have indeed concluded that the combination of universal tumor testing followed by cascade screening of relatives of probands diagnosed with LS appears reasonably cost-effective relative to no screening for LS in newly diagnosed patients with colorectal cancer (CRC). That does not mean, though, that universal tumor testing has been shown to be cost-effective vis-à-vis either age-targeted or selective screening.

Di Marco et al. cited two studies as concluding that universal testing was acceptable in cost-effectiveness terms relative to age-targeted testing. First, reference 30 reported an ICER of $\$ 37,010$ per life-year gained (LYG) for universal versus age-targeted testing in 2008 US dollars, but the ICER estimates in that article were later acknowledged by the authors to have been understated by about $40 \%$. $^{3}$ Applying the same 1.4 ratio to the ICER for universal versus agetargeted testing yields an estimate of \$52,211 in 2008 US dollars, which is above the $\$ 50,000$ benchmark. Also, if outcomes were expressed in quality-adjusted life-years (QALYs) rather than life-years, the ICER would have been roughly $20 \%$ higher than that figure.

Di Marco et al. cite a Canadian study (https://www.ncbi. nlm.nih.gov/pubmed/26468565) as finding that universal tumor testing in patients with CRC would be cost-effective relative to testing patients under age 70 . However, the authors of that study acknowledged that their ICER estimates were low because the cost of tumor testing was assumed to be low, about $1 / 3$ to $1 / 5$ as high as in other studies. ${ }^{2}$ If tumor testing is truly inexpensive, universal tumor testing could be cost-effective if even a handful of additional cases are detected.

Di Marco et al. did not report ICER estimates for the comparison of universal tumor testing relative to age-targeted testing that exceeded the conventional $\$ 50,000$ per LY or QALY benchmark. In particular, reference 33 reported that universal testing would cost $\$ 88,700$ per LYG in 2010 US dollars relative to testing patients under age 70 . That was equivalent to roughly $\$ 100,000$ per LYG in 2015 US dollars. ${ }^{2}$

Some experts have suggested that testing patients under age 70 years may be considered more cost-effective relative to universal tumor testing. I previously noted that "more work is needed to assess the incremental cost-effectiveness of universal testing relative to testing with an age cutoff of 70". 2 Whether additional studies to answer that question would be helpful depends on whether decision makers would use that information, which is uncertain. US clinical guidelines call for either universal testing for LS in patients with CRC or testing of all patients under age 70 together with selective testing of patients over age 70 based on family history data. Because universal testing is considerably simpler to implement, the comparison of universal and targeted LS testing strategies may no longer be a question of active interest in most US settings. Healthcare systems that are more cost-conscious may choose differently. Context matters. 


\section{ACKNOWLEDGEMENTS}

The findings and conclusions in this report are those of the authors and do not necessarily represent the official position of the Centers for Disease Control and Prevention.

\section{DISCLOSURE}

The author declares no conflicts of interest.

Scott D. Grosse, $P h D^{1}$

${ }^{1}$ National Center on Birth Defects and Developmental Disabilities, Centers for Disease Control and Prevention, Atlanta, Georgia. Correspondence: Scott D. Grosse (sgrosse@cdc.gov)

\section{REFERENCES}

1. Di Marco M, D'Andrea $E$, Panic $N$, et al. Which Lynch syndrome screening programs could be implemented in the "real world"? A systematic review of economic evaluations. Genet Med. 2018; doi:10.1038/gim.2017.244.

2. Grosse SD. When is genomic testing cost-effective? Testing for Lynch syndrome in patients with newly-diagnosed colorectal cancer and their relatives. Healthcare (Basel). 3:860-878; 2015.

3. Grosse SD, Palomaki GE, Mvundura M, Hampel H. The cost-effectiveness of routine testing for Lynch syndrome in newly-diagnosed patients with colorectal cancer in the United States: corrected estimates. Genet Med. 17:510-511; 2015.

Advance online publication $\mathrm{xx}$ xx 2018. doi:10.1038/s41436-018-0025-5 